\title{
An Efficient Hierarchical Representation Approach of Remote Sensing Application Modeling Based on Distributed Environment
}

\author{
Quan Zou (D), ${ }^{1}$ Wenyang Yu ${ }^{D}{ }^{2}$ and Guoqing $\mathrm{Li}^{2}$ \\ ${ }^{1}$ School of Computer Information and Science, Centre for Research and Innovation in Software Engineering, \\ Southwest University, No. 2 Tiansheng Road, BeiBei District, Chongqing 400715, China \\ ${ }^{2}$ Aerospace Information Research Institute, Chinese Academy of Sciences, Beijing 100094, China \\ Correspondence should be addressed to Wenyang Yu; yuwy@radi.ac.cn
}

Received 3 August 2020; Revised 15 October 2020; Accepted 16 November 2020; Published 3 December 2020

Academic Editor: Emilio Insfran Pelozo

Copyright (C) 2020 Quan Zou et al. This is an open access article distributed under the Creative Commons Attribution License, which permits unrestricted use, distribution, and reproduction in any medium, provided the original work is properly cited.

In Earth science, information science, space science, and other disciplines, scientists use the land surface parameter inversion method in their work, applying this to the atmosphere, vegetation, soil, drought, and so on. Multidisciplinary experts sometimes collaborate on a particular application. However, these remote sensing models do not have a unified method of description and management and cannot effectively achieve the sharing of models and data resources. It is also hard to meet user demand for global data and models in the current state, especially in the face of global problems and long-term series problems. In this paper, we examine the scientific questions of the computability and scalability of remote sensing models. This paper adopts a data dependency approach to describe a remote sensing model and implements a hierarchical unified description and management method using modelling based on four layers: a data-processing view, an atomic model view, an on-demand resource package view, and a workflow view. We choose three typical remote sensing models for disaster monitoring as use cases and describe the practical application process of the proposed method. The results demonstrate the advantages and powerful capabilities of this efficient method.

\section{Introduction}

The remote sensing model, also known as the remote sensing information model, is a model established by applying remote sensing information and geographic imaging methods $[1,2]$. The use of remote sensing technology to study the essence of the Earth involves simulating the surface landscape with various images, expressing the physical, chemical, and morphological attributes of the objects on the ground with spectral features. Remote sensing information is therefore a kind of mapping of ground objects, since there is a mathematical and geological correspondence between them. Scientists often use multiband remote sensing information to objectively describe ground objects or features and refer to this as a remote sensing model.

The data source of the remote sensing model is geoscience data, with a variety of auxiliary data acting as the driving data. The complex diversity of remote sensing models can be divided into three aspects: the data source, its function, and the implementation algorithm.

At present, global-scale issues such as atmospheric and environmental monitoring [3-5] and long-term sequence issues such as the comparative analysis of historical data to find specific rules for prediction [6-8] need to be supported by large-scale global data. Following the development of Earth observation technology, various industries and research institutes around the world have accumulated large amounts of spatial data and complex scientific models [9]. These data were received by ground stations and have been distributed to various regional data centres around the world for storage, forming an "information isolated island" phenomenon $[10,11]$, which has greatly restricted the development of spatial data sharing and applications. Accordingly, the issue of how to integrate and manage existing remote sensing model resources and data resources needs an urgent solution. It is difficult to effectively share 
and cooperatively process the different data and model information resources, meaning that the data provided may not be sufficient, and the required information may not be provided in a timely manner. Due to the differences in data content, data sources, data models, support platforms etc., the sharing of remote sensing models and data is challenging. In particular, with the mounting application requirements of spatial information data and models in various disciplines, fields, and industries, the need to share spatial data and scientific models is increasingly urgent, and it is also urgent to establish a new mechanism for the effective sharing and collaboration of different information resources. This mechanism must meet the requirements of fast, accurate, flexible, and comprehensive applications.

The development of remote sensing parameter inversion techniques has led to the establishment of many inversion models, such as snow parameters inversion model [12], forest reflectance inversion model [13], and surface water inversion model [14], that are used around the globe. There is a lack of correlation between these models, and their maximum potential cannot be achieved without proper management. The remote sensing model has characteristics of diversity and heterogeneity, making it difficult for users to autonomously organise and design the surface information processing flow to and allow it to run automatically. As a result of the lack of complete theoretical methods and mature technologies for unified representation, sharing, convergence, and efficient collaboration between various types of remote sensing information resources, it is difficult to accurately and quickly automate the organisation and management of data and model resources. In order to overcome the inefficiency in the sharing and interoperation of remote sensing models, there is an urgent need to provide new representation approaches for the increasing number of global users so that they can create remote sensing models more efficiently and more intelligently.

Existing methods of sharing remote sensing information primarily describe remote sensing information through various metadata standards and service standards and through publishing and discovery based on a registry to achieve information sharing $[15,16]$. Satellite remote sensing metadata describes data from satellite remote sensing, including satellite identification, sensor identification, and other remote sensing information, spatial projection, geodetic data, and other geographic information, and image types, image storage locations, and other image information [17]. Metadata provide an effective means of storage management and sharing of satellite remote sensing data. The use of metadata to establish data catalogues and data exchange centres for massive satellite remote sensing data offers the possibility of sharing spatial data.

Service standards include registration, processing, and application service standards. Processing services typically relate to remote sensing image processing, such as remote sensing image enhancement, cutting and mosaic, edge extraction, image classification, and others. Application services involve remote sensing applications [17], which use the collection of remote sensing data to investigate, monitor, analyze, predict, and forecast the Earth's resources, environments, and disasters. Services are provided with detailed descriptions and definitions in the form of service interfaces. A common approach is to establish a remote sensing data sharing service using web services $[18,19]$ and to adopt universal description, discovery, and integration (UDDI), and to register, publish, and discover various remote sensing information services [20]. Workflow technology combines multiple services in a certain order to complete complex remote sensing information sharing services simultaneously [21-23].

The Open Geospatial Consortium (OGC) has carried out in-depth research in the field of spatial information sharing and services and has developed a series of geospatial data interoperability specifications that provide a unified application framework for the design and development of geospatial information integration. These works also lay the foundation for research into intelligent service models with massive remote sensing data [24-27]. Using OGC's WFS (web feature service) and WMS (web mapping service), Zhang et al. solved two issues related to real-time spatial data sharing involving the heterogeneity of existing GIS systems and file-level data sharing systems over the web, which can accelerate time-critical applications to quickly acquire and integrate spatial data over the web [28]. On this basis, a framework was proposed based on the Geospatial Semantic Web [29]. This study aimed to examine the use of Geospatial Semantic Web technologies such as ontology to reduce spatial data duplication.

Today, geospatial data are ever-present. Collaborations involving geospatial data, for example, in modelling, data analysis, and visualisation, must still overcome the barriers arising from the need for specialised software and expertise, among other challenges. Song et al. created a geospatial data architecture for scientific collaborations. The outcome of this project can enable researchers and educators to share geospatial data and tools on the web and to build dynamic workflows connecting data and tools, without requiring GIS expertise [30].

Most of the traditional spatial information service systems based on database theory are aimed at solving problems related to the organisation, management, and application analysis of geological data. For example, an application for geospatial data was built and edited based on an open source web technology [31], and interfaces have been designed to process geospatial data based on the OGC standard and to store data in a GeoJSON format in MongoDB. However, there is currently no better way to properly support the matching of various data and applications among geosciences, collaborative services, and efficient processing in the field of remote sensing. This makes it difficult for geoscience data to be intelligently discovered by various practical applications and to be used effectively and reasonably. It is also difficult to support the increasingly complex demands for global applications involving surface information.

The remote sensing model based on scientific analysis closely links the application of remote sensing with geoscience data. In order to realise the collaborative services of remote sensing science, it is therefore necessary to share not 
only data and computing resources but also remote sensing model resources. In the same way as in data sharing research, researchers first attempted to study the sharing of models by defining model service metadata [32]. A "black box" was employed to publish heterogeneous remote sensing and GIS models to standard Web services for sharing and interoperation and then to integrate the remote sensing and GIS models using Web services, based on the geospatial workflow and semantic supported marching method [33]. Due to the complexity and disciplinary aspects of remote sensing models, various participants are needed to engage in the collaborative modelling process: model resources are provided by model providers, computing resources by computing resource providers, data resources by data resource providers, and the services of the published model are accessed by users. Most research in this area aims to provide a collaboration-oriented method through which modelling participants can conveniently work together and make full use of model, computing, and data resources across an open web environment. One of the fundamental concepts of HydroShare is that of a resource [34, 35]. All contents are represented using a resource data model, including different data types used in the hydrology community and models and workflows. A model service deployment strategy has also been proposed [36] by Wen et al., who presented a description method to help build model-deployment packages for model sharing.

Remote sensing information processing often involves multiple platforms and multiple sensors with distributed, multisource, and heterogeneous features, and this makes it difficult for other users to autonomously organise and design the surface information processing flow and to automate their simulation operations. In order to achieve effective sharing of remote sensing information, the most important aspect is the standardised and uniform representation of the model. A new mechanism is also needed to support ondemand description, management, and organisation. The significance of solving the above problems lies in allowing remote sensing models to be run automatically and implemented efficiently.

In geographic computing, workflows are often used to reduce the complexity of geographic information system application development, such as grid workflows shown in [37-40] for remote sensing applications. However, the development of remote sensing models requires a lot of prior knowledge, such as the architecture and workflow of grid or cloud computing systems, and it is still too complicated and difficult for most remote sensing application users. In order to facilitate modelling for scientists in the remote sensing field, this paper provides a model description method before the workflow design.

To make efficient use of a remote sensing model, it is important to collect or build an appropriate model resource and to describe a structure or schema to serve this purpose. The research in this paper focuses primarily on reducing the difficulties of describing a variety of remote sensing models, since an unclear description of these models can influence and hinder collaborative modelling studies. This paper examines the available approaches to describing model resource information. Based on the characteristics of remote sensing models, a model description strategy and a useroriented hierarchical representation system are designed. The basic goal of this description strategy is to provide modellers with a unified approach to describe models, to allow them to work together more conveniently, and to make full use of model resources in an open web environment.

The remainder of this paper is organised as follows. Section 2 explains how to design and implement description strategies. The procedure includes several different steps and measures, from the conceptual model to computation workflow for remote sensing models, and presents a useroriented hierarchical representation architecture for remote sensing models. A prototype is presented in Section 3, and it verifies that this method is practical and effective for the application process, based on some representative case studies. Final conclusions and plans for future work are given in Section 4, and a comparative analysis of the results is given.

\section{Methodology}

2.1. Model Description Framework. The core task of the model description framework is to provide a simple and convenient resource view and basis for description, for scientists in the field of remote sensing applications. Description and management of the data and processing for the model resources is achieved via a kind of "divide and rule" approach, which decomposes a large, complicated problem into multiple smaller problems.

The model description framework is task-oriented; the task is the basic component of the model, which is the entity that describes the model function and the execution process. In an on-demand description model, the task might be an image preprocessing algorithm, a physical model, a numerical calculation analysis, or even a complete remote sensing model. More specifically, in this paper, we define an on-demand description model as containing atomic tasks and composite tasks:

$$
\begin{aligned}
\text { Model }= & \{m \mid m \in \text { Atomic_task } \vee m \\
& \in \text { Composite_task }\} .
\end{aligned}
$$

Atomic_task: this paper defines the model task as one that is fully functional and cannot be divided into atomic tasks, and the intermediate result in the process is not used by others. In other words, an atomic task cannot contain subtasks, nor be subdivided using a higher granularity.

Composite_task: unlike an atomic task, a composite task is composed of several relatively independent and fully functional atomic tasks; this is a description of the process and function of the remote sensing parameters at different granularities.

We adopt the concept of resources to describe the task [41]. These resources primarily include data and processing resources, referred to here as "data" and "processing." In this paper, the remote sensing model is described by data-processing combinations; these two resources form the basic 
elements used in the remote sensing model. The term "data" refers to any structure, format, and amount of data that can be accessed and processed, while "processing" refers to a process that is imposed on certain data to generate new data (results).

In the remote sensing model, all applications can be described as a combination of data and processing, and this is known as a data dependency model. The data dependency model takes data and processing as the basic components, combines them into an application model, and provides a scheduling strategy for the application model. The processing order of the data-driven computing structure is determined by the interdependencies between the data and the validity of the operands. The data are connected between processes, and the time and space required for each process can be predetermined. As long as the data needed by the next step arrive and are useful, processing steps can be carried out to effectively exploit the temporal and spatial parallelism of the processing; consequently, the remote sensing model described by the data dependency model is very suitable for parallel processing.

Some of the core concepts in the data dependency model are defined as follows:

(i) Data: these may be basic data or a complex dataset. Denoted as $D$, they are represented by a circle in the data dependency model. A complex dataset is a set of datasets with the same type. It can be expressed as $D=\left\{D_{1}, D_{2}, D_{3}, \ldots, D_{n}\right\}$.

(ii) Processing: a set of output result data is obtained by processing a set of input data. This is denoted as $P$ and is represented by a rectangular box in the data dependency model. The processed input data is expressed as input $(P)$, which represents the input data or datasets needed by the processing $P$. The output data is expressed as output $(P)$, which represents the output data or datasets generated by the processing $\mathrm{P}$. And input $(P)$ and output $(P)$ are represented in $P$, corresponding to the value of Data $D$, respectively. The processing $\mathrm{P}$ is therefore expressed as $\{$ input, output, $\longrightarrow, P$, time $\}$, while input: $D_{\text {in }} \longrightarrow P$ ouput: $D_{\text {out }}$ represents the data input through the processing of $P$ to get the output data, that is, $D_{\text {out }}$ depends on $D_{\text {in }}$. $D_{\text {in }}$ is the input data value, and $D_{\text {out }}$ is the output data value. The variable Time represents the estimated processing time, which is used for task scheduling in distributed computing.

(iii) Processing should be limited in terms of time, and this time is recorded as Time $(\mathrm{P})$. If processing will not end within a limited time, the process is invalid or ill-conditioned.

(iv) Model: if the model as a whole is regarded as a task, regardless of the specific process, then this model represents the process of obtaining new data after a series of processing of the input data; during this process, a large amount of intermediate data will be produced. A remote sensing model is described using a detailed description, represented by a directed graph based on a data dependency, which is called the data dependency graph (DDG) of the model. This directed graph describes all the data and processing and clarifies the ways in which they interdepend. Consequently, the DDG describes how a model evolves from the initial dataset until the resulting dataset is obtained.

Hence, the model is formally represented as

$$
\text { Model }=\left(D_{s}, D_{i}, P, D_{t}\right) \text {. }
$$

In equation (2), $D_{s}$ is the original dataset, $D_{i}$ is the temporary result datasets $\left(D_{i} \in D, i=1,2, \ldots, m\right), P$ is the processing set, and $D_{t}$ is the target dataset.

The relationship between the processing and the data in the model is represented by the data dependency, as shown in Figure 1. For $\forall P_{j} \in P,(j=1,2, \ldots, n)$, if $\exists D_{i} \in \operatorname{input}\left(P_{j}\right)$, then there is a directed edge connection between $D_{i}$ and $P_{j}$ in the DDG of the model. If $\exists D_{i} \in \operatorname{output}\left(P_{j}\right)$, then there is a directed edge connection between $P_{j}$ and $D_{i}$ in the DDG of the model. This approach is used to connect the data and processing stages, and the DDG of the model is obtained in this way. In the DDG, the starting data $D_{s}$ are the data to which no processing is connected, that is, $\forall P_{j}, D_{s} \notin$ output $\left(P_{j}\right)$. The target data $D_{t}$ are data that are not directed to any processing; that is, $\forall P_{j}, D_{s} \notin \operatorname{input}\left(P_{j}\right)$. From Figure 1 , it can be seen that $D_{s}$ represents a data node without a predecessor node, and $D_{t}$ represents a data node with no successor node.

2.2. The Atomic Models. For a remote sensing model with data-intensive features, the emphasis of its description is on the selection and transfer of the processing resources, and thus the processing resources must be standardised. For each of the processing resources, it is necessary to provide information on the relevant data, such as its storage location and the scheduling approach use. A standardised processing resource can be measured in terms of its spatial and time complexity. The processing time of each task and the minimum path in the DDG map can be estimated, meaning that the model can be mobilised effectively for the resource. A remote sensing model requires multiple tasks to be combined, and in order to facilitate the conversion of the model, it is necessary to subdivide the model as far as possible. In this paper, we refer to the most subordinate task of the processing as an atomic model.

2.2.1. Concept. The atomic model is a nonsubdivided model, and we use the DDG to describe this, as shown in Figure 2.

The atomic model is formally represented as equation (3), and these meanings of $D_{s}, P$, and $D_{t}$ are the same as in equation (2):

$$
\text { Model }=\left(D_{s}, P, D_{t}\right) .
$$

The process of decomposing the remote sensing model into the atomic model must follow certain principles: 


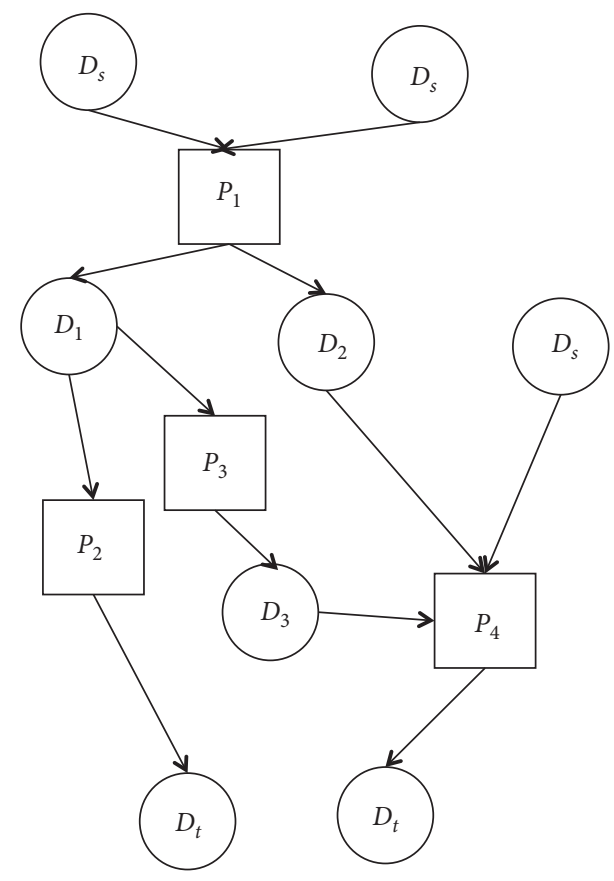

Figure 1: Data dependency model.

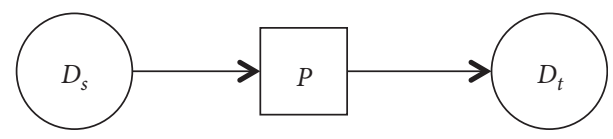

FIGURE 2: DDG of the atomic model.

(1) The principle of completeness, in which each atomic model is a complete task with all the elements of the data dependency model and has its own data-processing diagram.

(2) The principle of independence, in which each atomic model is independently executed as a task.

(3) The principle of hierarchical decomposition, in which a model is decomposed into multiple tasks, and each task is decomposed into multiple subtasks, and these are finally decomposed into atomic tasks (atomic models). The hierarchical decomposition clearly indicates the parent-child relationship of the task.

(4) The principle of loose coupling, in which atomic models are coupled as little as possible. In general, there is no need to know the specific processes of other atomic models.

A task is a basic component of the on-demand description model, and an atomic task is a model task that is fully functional and undecomposable. Functional integrity means that the atomic task must be able to complete a processing function in its entirety to produce a data product. An undecomposable task is a partition with the highest possible level of granularity, which does not contain subtasks. In the previous section, it was shown that each task is described by data and processing resources in the description of the remote sensing model. If a task has a subtask, the output data of this subtask is independent and meaningful. However, since an atomic task does not contain subtasks, the intermediate results generated by an atomic task are unable to be used by other tasks. An atomic task is also known as an atomic model of the remote sensing model, as it is the smallest component.

2.2.2. Representation of the Atomic Models. Generally, the remote sensing models are classified into three types, physical models (such as atmospheric correction), empirical models (such as interpretation of signs), and statistical models (such as supervised classification) [1]. According to specific applications, remote sensing models can be divided into agriculture models, forestry models, land models, disasters monitoring models, etc. [2]. Radu et al. classified remote sensing model algorithms into eight types according to the number of input images and the type of processing function [38]. And we summarized these computational characteristics and classified them into five categories [42]. Unlike the common application-based model classification, which represents the level of the data products and the production process of each level of these products, in this paper, the common remote sensing model is divided into five different types according to the calculation characteristics: the numerical, iterative, statistical, neighborhood, and frequency domain calculations. An atomic model in a remote sensing model will be classified according to a computational characteristic classification method and then represented by different calculation modes. (1) Numerical calculation. Numerical calculation means that the original data is added, subtracted, multiplied, divided, multiplied, exponent, logarithm, etc. at the pixel level. Typical examples are contrast changes, gradation transformations, and vegetation index calculations. (2) Iterative calculation. The iterative method is a typical method in numerical calculation. It is used to solve the roots of the equations, solve the equations, find the eigenvalues of the matrix, and so on. (3) Statistical analysis. In the statistical analysis of remote sensing application, it includes counting the data characteristics of each band or each type of the spectrum and doing the multivariate statistics between each band or each time series. (4) Neighborhood operation. Neighboring computing is the demand of the computing process for space dependence, which means that each pixel in the output image is determined by the corresponding input pixel and other pixels in a neighborhood. Some of the more typical neighborhood calculations are convolution, filtering, and so on. (5) Frequency domain operation. When the image is analyzed or processed in the frequency domain, it is usually necessary to first convert the time domain data into frequency domain data.

The atomic model is the object of all modelling information making up the remote sensing model. It captures the significant information and concepts related to modelling information and illustrates the relationship between them.

The conceptual framework of the atomic model defines the entity object of the model in order to maintain interoperability between atomic models and allows for easy 
indexing and finding of the atomic model. In this paper, we abstract the metadata describing the common features and relationships of the remote sensing model information, in order to provide a critical descriptive factor for unified model representation.

The most basic metadata information describing the atomic model consists of three elements (Figure 3):

(i) Model service metadata (ModelMetadata): this describes the metadata information about the data resources contained in the atomic model, and the Property object of DataMetadata indicates whether these are input or output data

(ii) Data resource metadata (DataMetadata): this describes the metadata information of the processing resources

(iii) Processing resource metadata (ProcessMetadata): this describes the information about the model service itself

\subsection{A User-Oriented Hierarchical Representation Architecture} for Remote Sensing Models. The resource package is a method for describing resources, as proposed by Zou et al. [41], and the model resources are encapsulated using the RDF method. A set of normative specifications was designed to describe abstract remote sensing models, including objects, relationships, operations, and rules.

Using the concept of on-demand service, the process of the remote sensing model is described by means of a resource-oriented package [41] that facilitates on-demand matching, searching, and access to distributed models. Based on the resource package model description method, this paper proposes a hierarchical representation architecture for realising simulations and descriptions of remote sensing models. The mechanism uses the definitions of the structure and organisation of the resource package, combines the model metadata information, and uses a formalised abstract model description to complete the standardised definition of the model.

In processing services for remote sensing models, a complex remote sensing model can be abstracted into a collaborative workflow of computing resources and data resources, and a process of automation and simulation can be realised, from data to information to knowledge.

In order to facilitate the use of a unified description approach to model resources, we design a hierarchical description mechanism for remote sensing models. The concept of a layered view is used to describe the mapping and implementation of the remote sensing model, from the conceptual model to the computer executable model, using a layer-by-layer approach. It is divided into four levels: a dataprocessing view, an atomic model view, an on-demand resource package view, and a workflow view. The overall process is shown in Figure 4.

Data-process view layer: in this layer, the DDG is used to describe a remote sensing model, abstracting it as a conceptual model. The specific approaches used here involve abstracting this complex remote sensing model into a simple combination of data and processing as elements and using a specific data-processing model to describe the user's desired remote sensing model. The DDG clearly expresses the dependency relationship between data and processing in a remote sensing model so that the remote sensing model can be described on an as-needed basis. The model described in this way is easy to be reused, and it is also convenient for scientists to perform distributed task processing and scheduling of a model in an open web environment.

Atomic model view layer: in this layer, the specific atomic model in the data-processing conceptual model in the previous layer is abstracted out and described. The granularity of each atomic model is described based on the classification of the computing features, including the representation of the calculation model and the abstraction of the data model. The metadata information of each atomic model are standardised to facilitate access and interoperability among the atomic models.

On-demand resource view layer: this layer regards all processing, data, and services in the remote sensing model as resources. Based on the definition of the resource package, the atomic models in the upper layer are encapsulated as resource packages. Consequently, a complete remote sensing model is described by a combination of specific data resource packages and computing resource packages.

In the actual geoscience application, the user creates a new remote sensing model using the defined resource package. Based on the description of the previous layer, users combine the metadata information of the atomic model defined in the previous step to select appropriate data and computing resources. A global view of on-demand resource packages is used to describe all the basic resources in the model and their relationships with each other, and the entire model is gradually constructed on this basis. The order relationships and interface matching of these resource services in the Earth model are described in this layer.

Workflow view layer: the above three layers of description are based on the user-oriented language. Before processing the remote sensing model described by the user, it is also necessary to convert the description of the upperlevel resource flow into a description of the final executable computer language flow.

For most of the existing remote sensing models, according to the perspective of the end user, the description and classification of the model can be divided into the aspects of the purpose of the model and the resulting dataset. However, computer programmers are likely to pay more attention to the implementation of the model, the specific operating platform, the implementation process, etc. Thus, these two types of operators have a different focus, and an urgent issue arises involving how to describe and manage the model so that it not only meets the specifications of the computer language but also shields the user from the specific details of the implementation of the platform, the programming language used, etc. We have solved the second of these problems through the first three layers of description, but the first needs to be solved in this layer. Models that are appropriate to the actual computing environment, such as distributed computing, grid computing, and cloud 


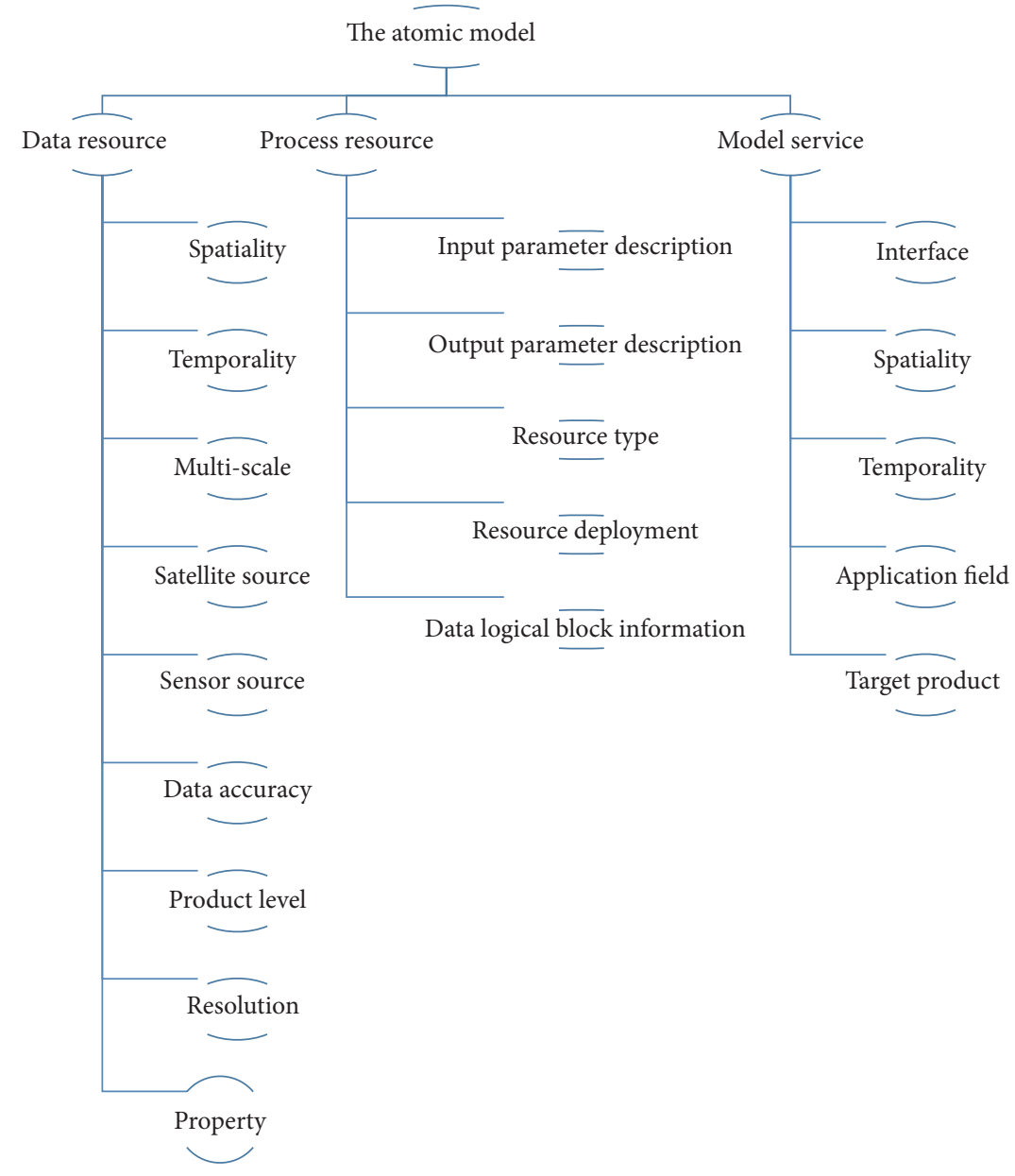

Figure 3: Metadata of the atomic model.

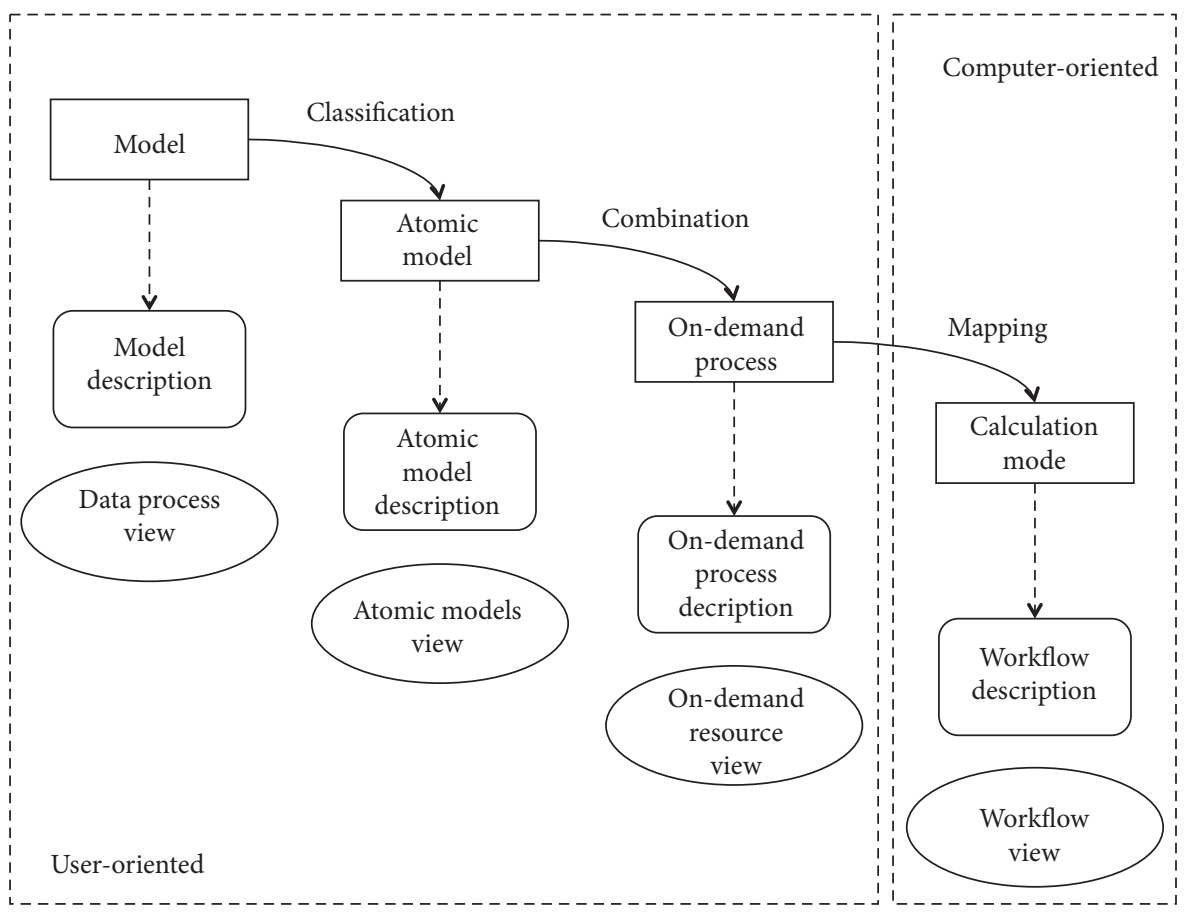

FigURE 4: Hierarchical mapping and implementation of a remote sensing model, from a conceptual model to a computer executable model. 
computing modes, are selected in this layer to describe the specific model. The description of the resource flow in the upper layer will provide a direct basis for the final formation of an executable model processing workflow at this layer.

Following this hierarchical mapping approach, a remote sensing model can be abstracted from the concept-based representation into an executable computer language representation in a step-by-step way. The user can describe the remote sensing model using this hierarchical mapping approach, thus facilitating shared access to and operation of the resources in the model and collaborative processing of the model.

\section{Results and Discussion}

Using the description method described in Section 2, this section first presents a vegetation drought monitoring model as an example to explain the hierarchical mapping proposed here for model sharing. Then, two other typical remote sensing models are examined as application cases: an aerosol optical thickness inversion model and a sand dust monitoring model. Based on these three examples, we provide a demonstration of various application paradigms. This study validates the standardisation, intelligence, and extensibility of our hierarchical representation of remote sensing models.

3.1. Examples of Vegetation Drought Monitoring Models. We use the data monitored via satellite remote sensing to calculate the vegetation index; if the index is found to be significantly reduced, this indicates that a drought has occurred. This is known as the vegetation index method for monitoring drought.

3.1.1. Model Introduction. In order to more effectively extract the moisture content of the vegetation canopy, Gao et al. [43] proposed the normal differential water index (NDWI) to monitor the drought situation in 1996. In this paper, a model for drought monitoring was established based on the anomaly water index (AWI) [44], which uses the relationship between the NDWI index and short-term climate change to monitor drought conditions for a wide range of vegetation crops. Since the main algorithm of this model is the AWI index calculation, it is referred to here as the AWI model.

The AWI model uses MOD09A1 data, which provides bands 1-7 at a resolution of $500 \mathrm{~m}$ in an eight-day gridded level-three product in the sinusoidal projection by NASA as input images. Each group of images undergoes NDWI and AWI calculation, colorisation, and visualisation to give the global range of droughts in each period.

The details of the processing are as follows:

(a) Extract the grayscale values of bands 2 and 6 in the input images.

(b) Calculate the NDWI value using the grayscale values of bands 2 and 6, as shown in equation (4); these values are represented by $\rho 2$ and $\rho 6$, respectively:

$$
\mathrm{NDWI}=\frac{\left(\rho_{2}-\rho_{6}\right)}{\left(\rho_{2}+\rho_{6}\right)}
$$

(c) Calculate the AWI value of each scene image. This calculation requires both the NDWI calculated in step 2 and the average value of the NDWI (AvgNDWI or $\overline{\mathrm{NDWI}}$ ) of the scene image on that date in previous years. If AvgNDWI already exists, this is calculated directly; if there is no AvgNDWI value yet for this area, this must be calculated before calculating AWI. In equations (5) and (6), $\mathrm{NDWI}_{\mathrm{i}}$ is the value of NDWI for the $i$ th year:

$$
\begin{gathered}
\overline{\mathrm{NDWI}}=\frac{\left(\sum_{i=\text { year }}^{\text {year }+10} \mathrm{NDWI}_{i}\right)}{11}, \\
\mathrm{AWI}=\mathrm{NDWI}_{i}-\overline{\mathrm{NDWI}} .
\end{gathered}
$$

(d) Mosaic multiple images.

(e) Colourise the result image according to the degree of drought represented by the AWI value.

(f) Visualise the resultant images as the output of the model.

3.1.2. Hierarchical Representation Approach for the AWI Model. Based on the hierarchical mapping method put forward in this paper, we can explain the description and simulation process of the remote sensing information model as follows.

(1) Data-Process View. In the data-processing view layer, the data and processing task tree is used to describe the AWI model on-demand (using the $n$-view input image as an example). The processing in the model is refined into a combination of atomic models. By abstracting it as a conceptual model, a complex AWI model is transformed into a simple combination of data and processing elements, thus clearly expressing the dependencies between data and processing.

In this data dependency model (Figure 5), the processing includes band selection, calculation of NDWI, AWI and AvgNDWI, format conversion, multiple image mosaics, and visualisation. Since these are atomic models, they can be used directly by other models.

(2) Atomic Model View. The atomic model of the AWI model is shown in Table 1, based on the decomposition principles of the atomic model defined in Section 2.2.1. Using the classification of computational characteristics ((1) numerical calculation, (2) iterative calculation, (3) statistical analysis, (4) neighborhood operation, and (5) frequency domain operation), the categories of these atomic models are shown in Table 1. We then describe the granularity of each atomic model, including the expression of the computational model and the abstraction of the data model, and normalise the metadata information of each atomic model to facilitate access and interoperability between atomic models. 


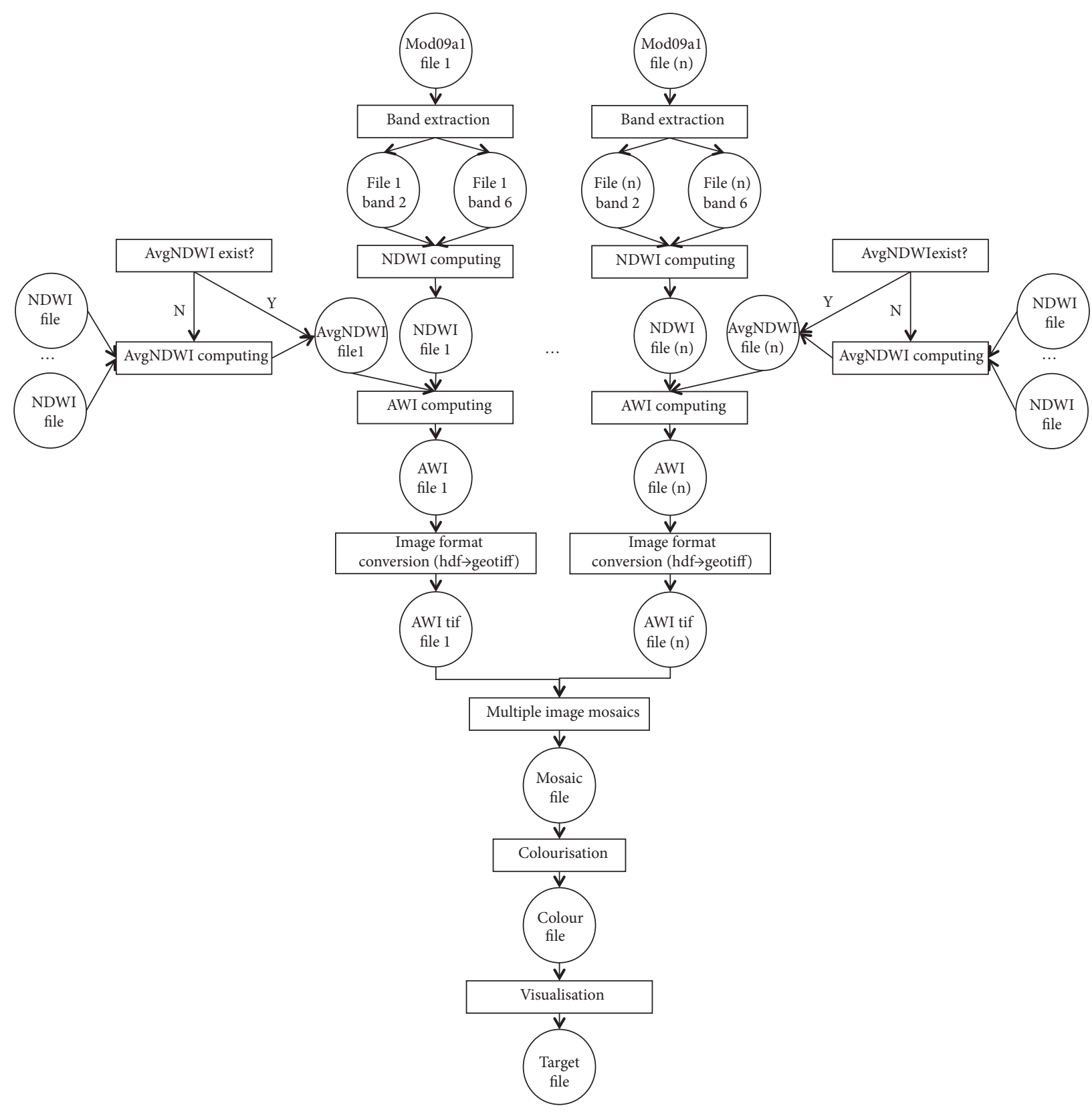

FIGURE 5: DDG of the drought monitoring model.

TABLE 1: Classification of computational characteristics of atomic models in AWI models.

\begin{tabular}{lccccccc}
\hline Atomic model & Band selection & NDWI & AWI & AvgNDWI & Format conversion & Mosaic & Visualisation \\
\hline Classification & 1 & 1 & 1 & 1 land3 & 4 & 4 & 4 \\
\hline
\end{tabular}

(3) On-Demand Resource View. We define these atomic models using the concept of resource packages. Users can reuse previously defined resource packages, thus reusing model resources. Figure 6 illustrates the use of resource packages to describe the process representation of the AWI model.

Using the on-demand organisation approach based on the resource package, it can be relatively straightforward to implement a deployment configuration in a remote sensing model, and this is applicable to a variety of environments. The definition of a resource package is not limited to a computing environment; experiments show that the resource package approach is suitable for numerous environments and is configurable.

(4) Workflow View. In a distributed network computing platform such as a grid computing or cloud computing platform, all resources can be used and accessed by 


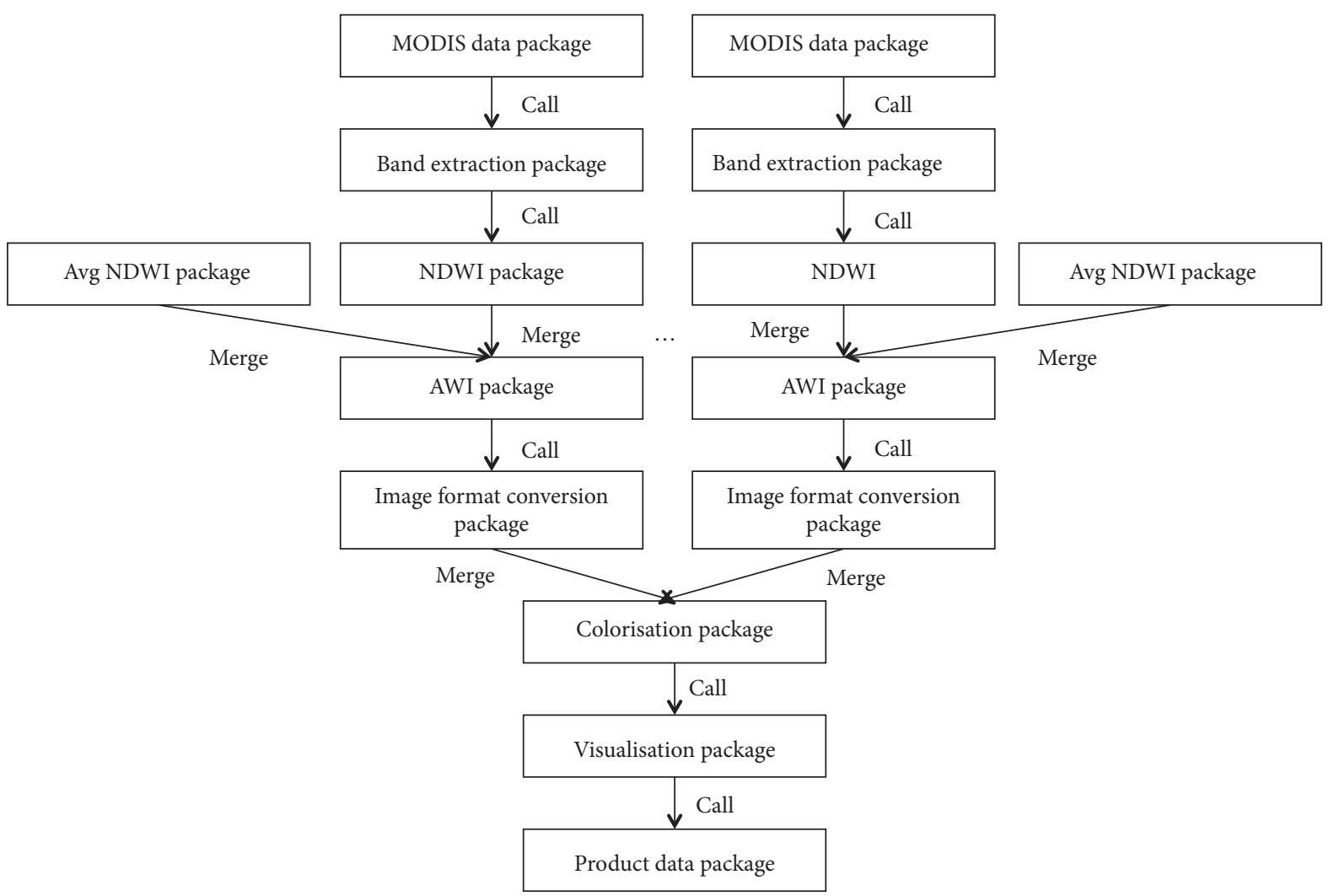

FIgure 6: Processing flow of the AWI model based on resource package definition.

encapsulated services such as Web services or Cloud services. Using the description of the processing flow in the ondemand resource view, we can obtain detailed information about the resource interface and describe it in a specific workflow, such as XML Process definition Language (XPDL), Business Process Execution Language (BPEL), and Resource Description Framework (RDF).

The workflow for the AWI model is shown in Figure 7. File 1 to File 8 mean eight raw remote sensing images. Execution of this can realise the operation and processing of the disaster monitoring model, using remote sensing data according to the user's needs, and can return the result to the user. The resultant data product therefore contains meaningful information.

We applied this user-oriented hierarchical description approach for remote sensing information models to largescale distributed computing prototype systems, thereby achieving the semiautomatic description, calculation, and sharing of multiple remote sensing models.

The current prototype system can generate workflows only semiautomatically based on the workflow description mode of each model. These modes are manually designed according to the workflow view description in our hierarchical model description approach. However, the value of this approach in enabling the sharing of remote sensing models is clear.

Using the method proposed in this article, these atomic models can be reused, which facilitates model sharing. And it can be parallelized and scheduled in an open web environment, especially when computing resources are scattered. This model expression method is proposed to facilitate the algorithm design of remote sensing scientists, and the designed DDG model is convenient for programmers to perform distributed computing programming and design workflow. In [45], we have experimented with this method. We implemented these use cases in the prototype system using cloud computing.

3.2. Use of the Remote Sensing Model for Disaster Monitoring. In our prototype system, we used the hierarchical description approach to create several typical disaster monitoring models as follows.

3.2.1. Dust Storm Monitoring Model. The details of the processing are as follows:

(a) Carry out geometric corrections for bands 1, 2, 5, 6, 7,20 , and 29 of the input images.

(b) Calibrate the corrected data.

(c) Extract dust information according to the reflectance of bands $1,2,5,6$, and 7 . The results will be used as a mask for dust classification based on differences in brightness temperature.

(d) Calculate the brightness temperature using the calibration results.

(e) Based on the differences in brightness temperature between bands 20 and 29, classify the dust storm at four levels. 


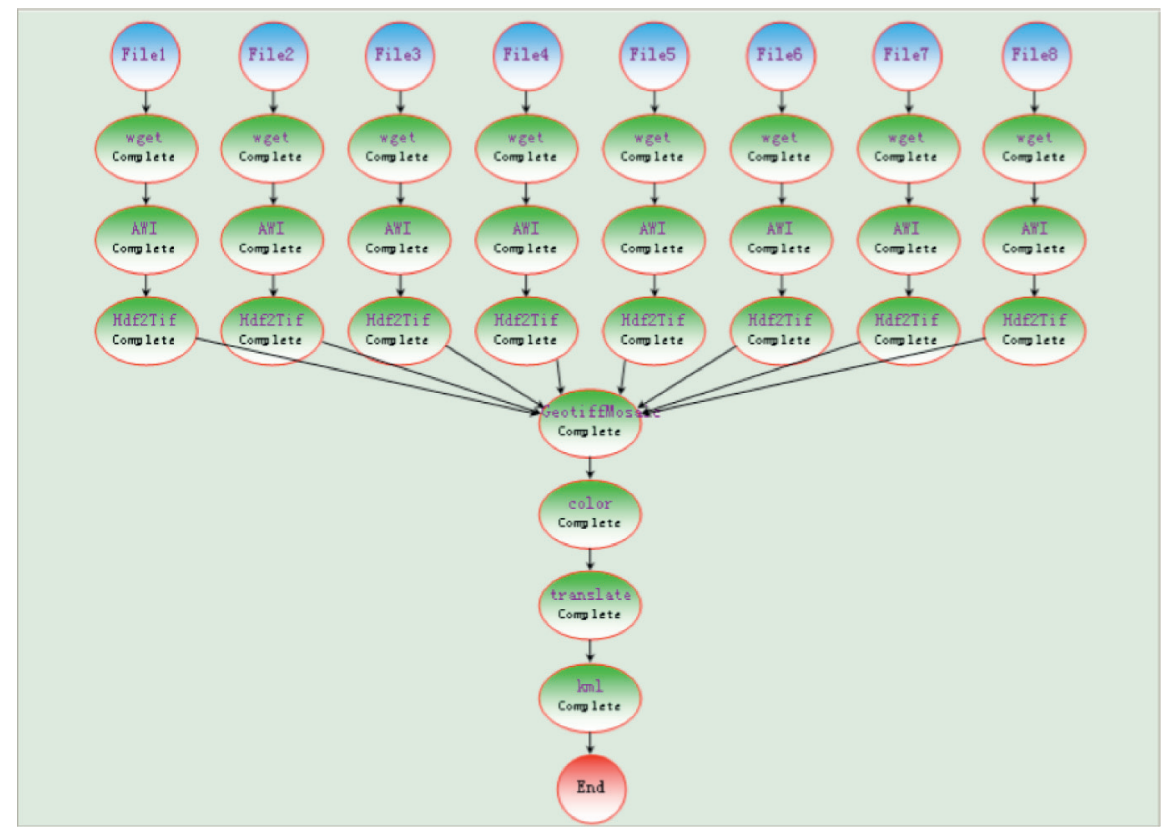

Figure 7: Workflow of the AWI model.

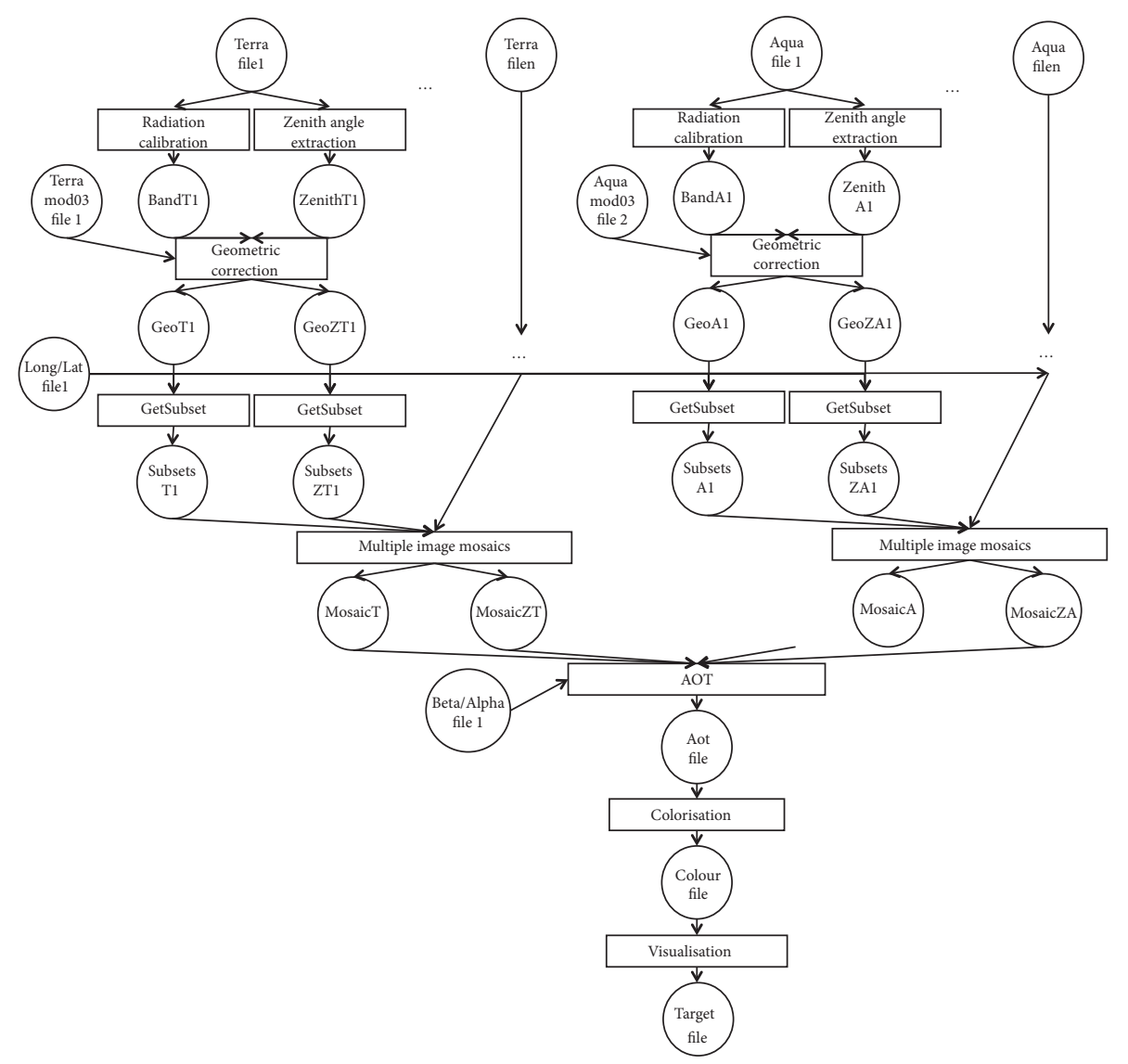

(a)

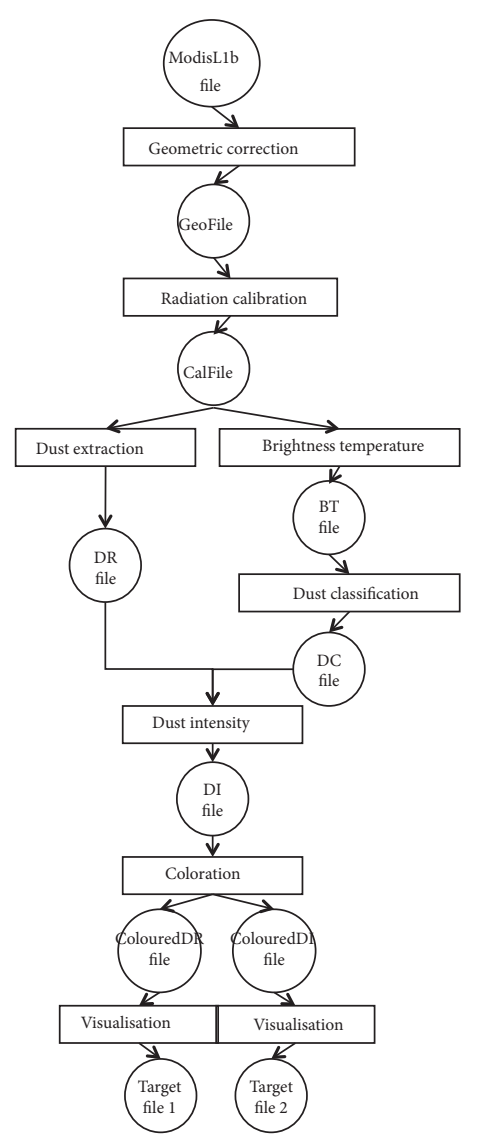

(b)

FIgURE 8: DDG of the AOT (a) and dust storm monitoring (b) models. 


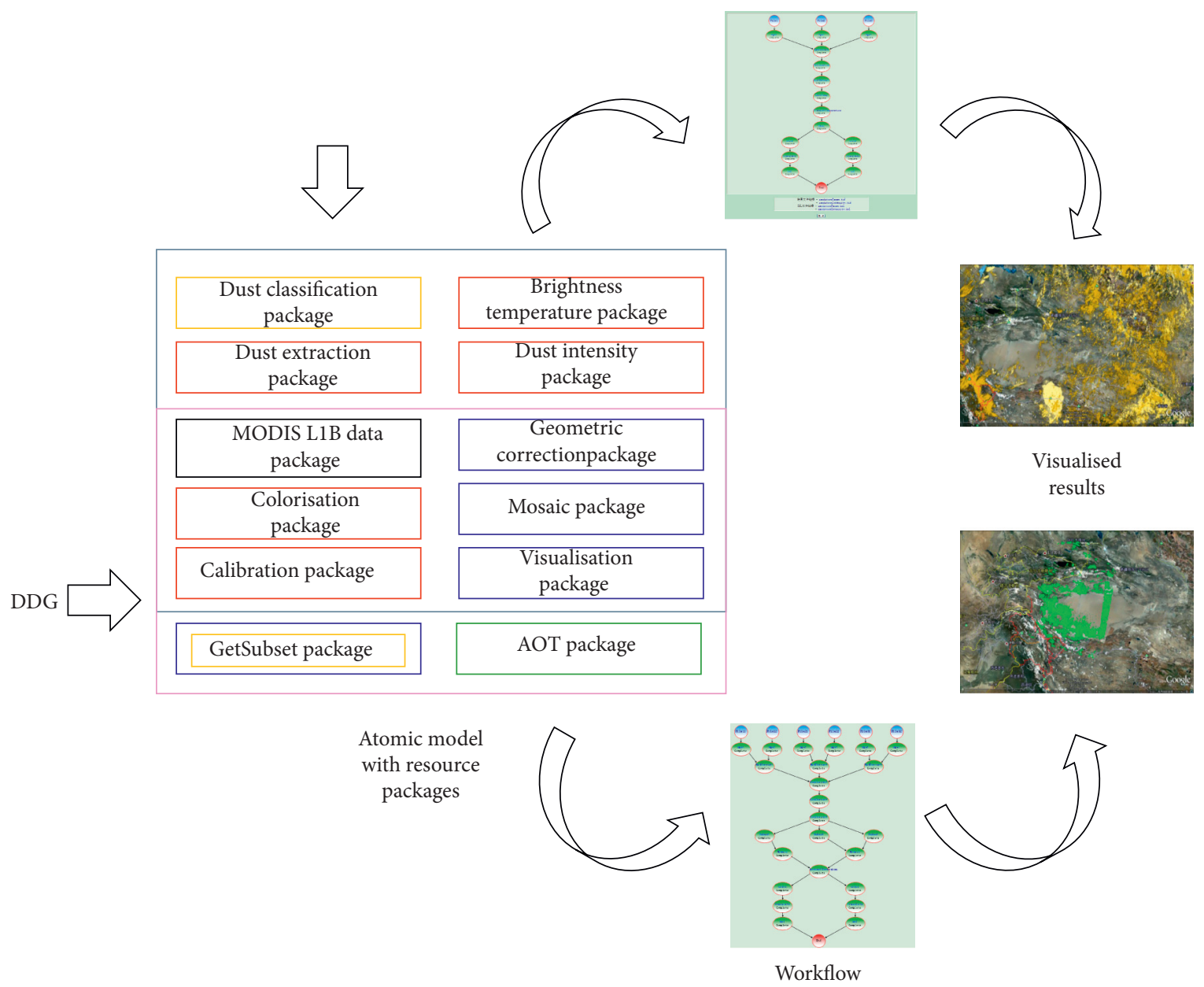

FIgURE 9: Hierarchical representation architecture of the AOT and dust storm monitoring models (in the atomic model with resource packages view; the red rectangle represents numerical calculation; the green rectangle represents iterative calculation; the yellow rectangle represents statistical analysis; and the blue rectangle represents neighborhood operation).

(f) Obtain the intensity of the dust storm using the extracted dust information as a mask.

(g) Colourise the resultant image according to four levels of intensity for the dust storm.

(h) Visualise the resultant images as the output of the model.

3.2.2. Aerosol Optical Thickness (AOT) Inversion Model. The details of the processing are as follows:

(a) Carry out radiometric calibrations for bands 1, 2, 3, 4,7 , and 10 of both TERRA and AQUA data

(b) Find the exact sensor zenith and solar zenith of both the TERRA and AQUA data

(c) For each band and zenith, carry out a geometric correction

(d) Capture subset data and mosaic for spatial range consistency of TERRA and AQUA data

(e) Colourise the resultant image according to AOT indexes

(f) Visualise the resultant images as the output of the model
Figures 8 and 9 show the application of the hierarchical description approach to these two models. It can be seen that, in the atom model view, the two models share several atomic models, as described by the resource packages, which can increase the reuse of resources and increase the speed of modelling. The data-processing view clearly illustrates the parallelisation of the atomic model, which provides a theoretical basis for the design of the workflow.

\section{Conclusions}

This paper selects three examples of descriptions of typical application models and demonstrates the practical application process of the proposed method. Using the approach proposed in this paper, instances of an AWI model, a dust monitoring model, and an aerosol monitoring model can be successfully described, confirming the advantages and power of the functions in describing and building complex models. The techniques presented in this paper significantly improve the reusability of remote sensing models, as shown in our case studies. Based on the experimental results, it is confirmed that the approach proposed in this paper can achieve expectations. The process of model description is simple and 
easy to understand, and the on-demand process does not require the user to have a high degree of expertise. Although there are still some deficiencies in the semantic recognition of user requirements in this approach, this unified model description provides a new direction for research into automated simulation of remote sensing models.

\section{Data Availability}

The remote sensing data used to support the findings of this study are available from the corresponding author upon request.

\section{Conflicts of Interest}

The authors declare no conflict of interest.

\section{Acknowledgments}

This study was supported by the International Partnership Program of Chinese Academy of Sciences, Grant no.131211KYSB20180002. The funders had no role in the design of the study, in the collection, analyses, or interpretation of data, in the writing of the manuscript, or in the decision to publish the results.

\section{References}

[1] C. Zhou, J. Luo, X. Yang et al., Remote Sensing Imaging and Analysis, Science Press, Beijing, China, 1999.

[2] A. Ma, Remote Sensing Information Model, Peking University Press, Beijing, China, 1997.

[3] R. S. DeFries and J. R. G. Townshend, "NDVI-derived land cover classifications at a global scale," International Journal of Remote Sensing, vol. 15, no. 17, pp. 3567-3586, 1994.

[4] B. Mueller and S. I. Seneviratne, "Hot days induced by precipitation deficits at the global scale," Proceedings of the National Academy of Sciences, vol. 109, no. 31, pp. 1239812403, 2012.

[5] E. R. Sholkovitz, P. N. Sedwick, T. M. Church, A. R. Baker, and C. F. Powell, "Fractional solubility of aerosol iron: synthesis of a global-scale data set," Geochimica et cosmochimica acta, vol. 89, pp. 173-189, 2012.

[6] M. E. Mann, R. S. Bradley, and M. K. Hughes, "Global-scale temperature patterns and climate forcing over the past six centuries," Nature, vol. 392, no. 6678, pp. 779-787, 1998.

[7] F. Bartolini, A. Barausse, H.-O. Pörtner, and F. Giomi, "Climate change reduces offspring fitness in littoral spawners: a study integrating organismic response and long-term timeseries," Global Change Biology, vol. 19, no. 2, pp. 373-386, 2013.

[8] F. V. D. Bergh, K. Wessels, S. Miteff et al., "HiTempo: a platform for time-series analysis of remote-sensing satellite data in a high-performance computing environment," International Journal of Remote Sensing, vol. 33, no. 15, pp. 4720-4740, 2012.

[9] L. Fusco, R. Cossu, and C. Retscher, "Open grid services for ENVISAT and earth observation applications," in High Performance Computing in Remote Sensing, A. J. Plaza and C. .,-I. Chang, Eds., pp. 237-280, Taylor \& Francis, New York, NY, USA, 2007.

[10] T. C. Wai, HKSAR geospatial information hub (GIH)-a common geospatial information platform and spatial data portal, China: FIG Working Week 2007, "Strategic Integration of Surveying Services".

[11] Y. Ding, Y. Fan, Z. Du et al., "An integrated geospatial information service system for disaster management in China," International Journal of Digital Earth, vol. 8, no. 11, pp. 918-945, 2015.

[12] L. Tsang, Z. Chen, S. Oh, R. J. Marks, and A. T. C. Chang, "Inversion of snow parameters from passive microwave remote sensing measurements by a neural network trained with a multiple scattering model," IEEE Transactions on Geoscience and Remote Sensing, vol. 30, no. 5, pp. 1015-1024, 1992.

[13] M. Schlerf and C. Atzberger, "Inversion of a forest reflectance model to estimate structural canopy variables from hyperspectral remote sensing data," Remote Sensing of Environment, vol. 100, no. 3, pp. 281-294, 2006.

[14] F. Wang, B. Zhou, X. Liu, G. Zhou, and K. Zhao, "Remotesensing inversion model of surface water suspended sediment concentration based on in situ measured spectrum in Hangzhou Bay, China," Environmental Earth Sciences, vol. 67, no. 6, pp. 1669-1677, 2012.

[15] Q. Dai, J. Liu, and S. Liu, "Key techniques of massive remote sensing data sharing," Computer Engineering, vol. 34, no. 6, pp. 283-254, 2008.

[16] L. Wei, S. Liu, and J. Wang, "Geo-spatial data sharing based on metadata catalog service," Remote Sensing Technology and Application, vol. 20, no. 6, pp. 615-619, 2005.

[17] Q. Zou, G. Li, and W. Yu, "MapReduce functions to remote sensing distributed data processing-global vegetation drought monitoring as example," Software Practice \& Experience, vol. 48, no. 7, pp. 1352-1367, 2017

[18] M. A. Popov, N. N. Kussul, S. A. Stankevich et al., "Web service for biodiversity estimation using remote sensing data," International Journal of Digital Earth, vol. 1, no. 4, pp. 367376, 2008.

[19] Y. Zeng, G. Li, L. Guo, and H. Huang, "An on-demand approach to build reusable, fast-responding spatial data services," IEEE Journal of Selected Topics in Applied Earth Observations and Remote Sensing, vol. 5, no. 6, pp. 1665-1677, 2012.

[20] Universal description discovery and integration. http:// www.uddi.xml.org/.

[21] Q. Zou, G. Li, and W. Yu, "Remote sensing model simulating compute under the network environment for large scale MODIS data," Mathematics in Practice and Theory, vol. 42, no. 17, 2012.

[22] L. Bigagli, M. Santoro, P. Mazzetti, and S. Nativi, "Architecture of a process broker for interoperable geospatial modeling on the web," ISPRS International Journal of GeoInformation, vol. 4, no. 2, pp. 647-660, 2015.

[23] Q. Zou, G. Li, and W. Yu, "An integrated disaster rapid cloud service platform using remote sensing data," in Proceedings of the IEEE International Geoscience and Remote Sensing Symposium, pp. 5221-5224, Fort Worth, TX, USA, July 2017.

[24] Open Geospatial Consortium, Web Coverage Service (WCS), Open Geospatial Consortium, Inc, Wayland, MA, USA, 2006, http://www.opengeospatialorg/standards/wcs.

[25] Open Geospatial Consortium, Web Map Service Implementation Specification, Open Geospatial Consortium, Inc, Wayland, MA, USA, 2004, http://www.opengeospatialorg/ standards/wms.

[26] Open Geospatial Consortium, OpenGIS ${ }^{\circledR}$ Web Processing Service, Open Geospatial Consortium, Inc, Wayland, MA, USA, 2005, http://www.opengeospatial.org/. 
[27] Open geospatial Consortium, OGC, Consortium. OpenGIS Reference Model, Open Geospatial Consortium, Inc, Wayland, MA, USA, 2003, http://www.openggis.org/docs/ 03-040.pdf.

[28] C. Zhang and W. Li, "The roles of web feature and web map services in real-time geospatial data sharing for time-critical applications," Cartography and Geographic Information Science, vol. 32, no. 4, pp. 269-283, 2005.

[29] C. Zhang, W. Li, and T. Zhao, "Geospatial data sharing based on geospatial semantic web technologies," Journal of Spatial Science, vol. 52, no. 2, pp. 35-49, 2007.

[30] C. X. Song, L. Zhao, L. L. Biehl, V. Merwade, and N. Villoria, GABBs: Cyberinfrastructure for Self-Service Geospatial Data Exploration, Computation, and Sharing, Agu Fall Meeting, San Francisco, CA, USA, 2016.

[31] S. Jun and S. Lee, "Prototype system for geospatial data building-sharing developed by utilizing open source web technology," Spatial Information Research, vol. 25, no. 5, pp. 725-733, 2017.

[32] M. Feng, S. G. Liu, and N. H. Euliss, "Distributed geospatial model sharing based on open interoperability standards," Journal of Remote Sensing, vol. 13, no. 6, pp. 1060-1073, 2009.

[33] Z. Chen, H. Lin, and M. Chen, "A framework for sharing and integrating remote sensing and GIS models based on web service," The Scientific World Journal, vol. 2014, no. 1, 13 pages, Article ID 354919, 2014.

[34] D. G. Tarboton, R. Idaszak, and J. S. Horsburgh, “A resource centric approach for advancing collaboration through hydrologic data and model sharing," in Proceedings of the 11th International Conference on Hydroinformatics (HIC 2014), New York, NY, USA, August 2014.

[35] D. G. Tarboton, R. Idaszak, and J. S. Horsburgh, "HydroShare: advancing collaboration through hydrologic data and model sharing," in Proceedings of the 7th International Congress on Environmental Modelling and Software, San Diego, CA, USA, June 2015.

[36] Y. Wen, M. Chen, S. Yue, P. Zheng, G. Peng, and G. Lu, “A model-service deployment strategy for collaboratively sharing geo-analysis models in an open web environment," International Journal of Digital Earth, vol. 10, no. 4, pp. 405-425, 2017.

[37] C. Nandra and D. Gorgan, "Defining earth data batch processing tasks by means of a flexible workflowdescription language," ISPRS Annals of Photogrammetry, Remote Sensing and Spatial Information Sciences, vol. III-4, pp. 59-66, 2016.

[38] A. Radu, V. Bacu, and D. Gorgan, "Diagrammatic description of satellite image processing workflow," in Proceedings of the Workshop on Grid Computing Applications Development (GridCAD) at the SYNASC Symposium, pp. 341-348, Timisoara, Romania, September 2007.

[39] D. Mihon, V. Colceriu, V. Bacu, and D. Gorgan, "Grid based processing of satellite images in Greenland platform," International Journal of Advanced Computer Science and Applications (IJACSA), vol. 3, no. 3, pp. 41-49, 2013.

[40] D. Mihon, V. Bacu, V. D. Colceriu, and D. Gorgan, "Modeling of earth observation use cases through the KEOPS system," in Proceedings of the Intelligent Computer Communication and Processing (ICCP), pp. 455-460, IEEE-Press, Cluj-Napoca, Romania, September 2015.

[41] Q. Zou, G. Li, W. Yu, and Y. Cao, "A "resource package"-oriented approach for remote sensing analysis modeling-dust storm monitoring model as example," International Journal of Software
Engineering and Knowledge Engineering, vol. 24, no. 05, pp. 731-757, 2014.

[42] Q. Zou, Q. Li, and W. Yu, "A model representation apporach based on computational characteristics for disaster monitoring," in Proceedings of the IEEE International Geoscience and Remote Sensing Symposium, Yokohama, Japan, August 2019.

[43] B.-C. Gao, "NDWI-A normalized difference water index for remote sensing of vegetation liquid water from space," Remote Sensing of Environment, vol. 58, no. 3, pp. 257-266, 1996.

[44] L. Bai, Q. Yan, and L. Zhang, "Sensitivity analysis of response of MODIS derived drought indices to drought in North China," Arid Land Geography, vol. 35, no. 5, pp. 708-716, 2012.

[45] Q. Zou, G. Li, and W. Yu, "Cloud computing based on computational characteristics for disaster monitoring," Applied Sciences, vol. 10, no. 19, p. 6676, 2020. 